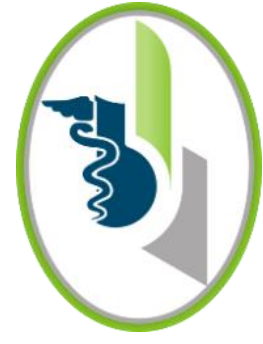

ACCESO ABIERTO

Para citaciones: Manrique, E., Palacio, B., Manotas, M., Rudas, M., Segura, J. (2019). Intoxicación paralítica por moluscos, revisión del tema. Revista Ciencias Biomédicas, $8(2), 87-94$.

Recibido: 27 de febrero de 2019 Aprobado: 23 de mayo de 2019

Autor de correspondencia: Elin Yohana Manrique Julio elinmanriqueuis@hotmail.com

Editor: Inés Benedetti. Universidad de Cartagena-Colombia.

Copyright: (C 2019. Manrique, E., Palacio, B., Manotas, M., Rudas, M., Segura, J. Este es un artículo de acceso abierto, distribuido bajo los términos de la licencia https://creativecommons.org/licenses/bync-sa/4.0/ la cual permite el uso sin restricciones, distribución y reproducción en cualquier medio, siempre y cuando el original, el autor y la fuente sean acreditados.

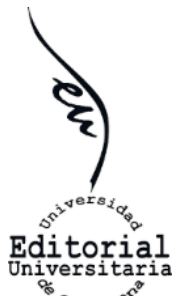

\section{Intoxicación paralítica por moluscos, revisión del tema}

\author{
Paralityc shellfish poisoning, a review.
}

\author{
Manrique-Julio Elin Yohana ${ }^{1}$, Palacio-Villalba Belkis ${ }^{1}$, Manotas-Castellar Marco Aurelio², \\ Rudas-Arrieta Margaret ${ }^{3}$, Segura-Posada Jhon Jairo ${ }^{4}$
}

${ }^{1}$ FATOXACOL, Barranquilla, Colombia

${ }^{2}$ Elanco animal health, Barranquilla, Colombia

${ }^{3}$ Viva IPS, Barranquilla, Colombia

${ }^{4}$ Clínica La Misericordia, Cartagena, Colombia

\section{RESUMEN}

Introducción: la intoxicación paralítica por moluscos es una enfermedad neurotóxica ocasionada por el consumo de moluscos contaminados por saxitoxina y sus derivados La neurotoxicidad se debe a la acción de la toxina sobre canales de calcio bloqueando la excitación neuromuscular. El tratamiento es sintomático, consiste en medidas de soporte hemodinámico y ventilatorio. En Colombia no se han reportado casos de intoxicación en humanos, pero en los últimos años el género Alexandrium ha sido detectado en muestras de agua provenientes de mareas rojas. Es necesario el monitoreo de las zonas de pesca o bancos de moluscos en Colombia para analizar la presencia de la biotoxina.

Objetivo: conocer el comportamiento de la saxitoxina en la intoxicación paralítica por moluscos.

Métodos: se realizó una revisión temática, para la cual se llevó a cabo una búsqueda electrónica en las bases de datos Sciencedirect y Scopus, y mediante el motor de búsqueda Google Académico, fueron adquiridos en texto completo, revisados y citados a lo largo de la presente revisión.

Resultados: se obtuvieron 50 artículos, de los cuales, 27 fueron considerados pertinentes, dichos estudios fueron adquiridos en texto completo, revisados y citados a lo largo de la presente revisión.

Conclusión: la intoxicación paralítica por moluscos puede afectar seriamente la salud humana, por tal motivo, y puesto que el género Alexandrium ha sido detectado en nuestros mares, se hace necesario el monitoreo de las aguas costeras donde se cultivan o pescan moluscos. Además, se hace necesario regular los límites permitidos de saxitoxinas en productos del mar.

Palabras Clave: Intoxicación por moluscos; saxitoxina; marea roja; floraciones algales nocivas.

\section{ABSTRACT}

Introduction: paralytic shellfish poisoning is a neurotoxic disease caused by the consumption of shellfish contaminated by saxitoxin and its derivatives. Neurotoxicity is due to the action of the toxin on calcium channels, blocking 
neuromuscular excitation. Treatment is symptomatic, consisting of hemodynamic and ventilatory support measures. In Colombia, no cases of poisoning have been reported in humans, but the genus Alexandrium has been detected in water samples from red tides in recent years. It is necessary to monitor freckle areas or mollusc banks in Colombia to analyze the presence of this biotoxin.

Objective: to know the behavior of saxitoxin in paralytic intoxication by molluscs.

Methods: thematic review. An electronic search was carried out in the Science direct, Scopus databases and through the Google Academic search engine.

Results: were obtained fifty articles, but only twentyseven abstracts were considered relevant. All studies were acquired in full text, reviewed and cited throughout the present review.

Conclusion: paralytic sellfish poisoning can seriously affects human health, and because the Alexandrium genus has been detected in our seas, it is necessary to monitor the coastal waters where sellfish are grown. In addition, it is necessary to regulate the permitted limits of saxitoxins in seafood.

Key Words: Shellfish poisoning; saxitoxin; red tide; harmful algal blooms.

\section{INTRODUCCIÓN}

Las floraciones de algas marinas producen toxinas paralizantes las cuales se acumulan en los tejidos de moluscos filtradores, conociéndose más de 20 neurotoxinas pertenecientes a este grupo, que son sintetizadas por los géneros de dinoflagelados autótrofos Gymnodinium, Alexandrium y Pyrodinium $(1,2)$, la más potente es la saxitoxina, producida por el género Alexandrium. Estas toxinas pueden ingresar al cuerpo humano por medio del consumo de mariscos contaminados, desencadenando una intoxicación paralítica, que es potencialmente tóxica (3). No solo se han detectado estas toxinas en organismos filtradores, sino también en no filtradores como cangrejos, langostas y caracoles (4). En cefalópodos como los pulpos se ha detectado saxitoxina en sus tentáculos, los cuales son de interés en la alimentación humana (2).

Las personas intoxicadas con toxinas paralíticas de moluscos, generalmente exhiben síntomas como: entumecimiento, debilidad muscular, ataxia, descoordinación motora, y disminución en la frecuencia respiratoria, siendo esta potencialmente fatal (5).
Las toxinas paralíticas de moluscos son un grupo de alcaloides neurotóxicos, la mayoría de estas toxinas son hidrófilas y de acuerdo con su cadena lateral pueden clasificarse como Nsulfocarbamoyl, carbamato y decarbamoyl. Recientemente se han detectados cadenas laterales con sustituyentes hidrófobos como hidrobenzoato (6).

En países como el Reino Unido y los Estados Unidos se controla de forma rutinaria la concentración de saxitoxina en la costa, esta no debe exceder el límite permitido de $80 \mu \mathrm{g}$ por cada $100 \mathrm{~g}$ de tejido de mariscos (7).

En Alaska entre los años 1973 y 1992 se presentaron 117 casos de intoxicación con toxinas paralíticas de moluscos., de los cuales una persona murió, cuatro necesitaron soporte ventilatorio con intubación, y 29 debieron ser trasladadas con urgencia a un hospital (8)

En Colombia no se han registrado casos de intoxicación paralítica por moluscos, pero algunos estudios recientes realizados en aguas de las mareas rojas ocurridas en el país, muestran la presencia del género Alexandrium $(9,10)$. Con esta 
revisión se busca conocer el comportamiento de la saxitoxina en la intoxicación paralítica por moluscos.

\section{METODOS}

Tipo de estudio: revisión temática y descriptiva en la que se incluyeron revisiones sistemáticas, investigaciones originales, editoriales y revisiones de tema.

Tipo de participación: documentos científicos sobre intoxicación paralítica por moluscos, mecanismo de acción de la toxina y manifestaciones clínicas.

Estrategia de búsqueda bibliográfica: se realizó búsqueda informática en inglés y español en las bases de datos ScienceDirect, Scopus y Google Académico. Las búsquedas se limitaron a los idiomas inglés y español, el período de revisión fue de 2010 a 2020.

Términos clave: fueron tomados los siguientes descriptores: Intoxicación por moluscos, saxitoxina, marea roja, floraciones algales nocivas, así como su respectiva traducción al inglés.

Métodos de revisión: se estudiaron títulos, resúmenes y textos completos de los documentos seleccionados en las bases de datos consideradas. Si los resúmenes se ajustaban a las estrategias del objetivo planteado, se obtuvieron los artículos completos.

Recopilación y análisis de datos: se revisaron todos los artículos completos de forma independiente.

\section{RESULTADOS}

Se consideraron cincuenta artículos publicados en diferentes revistas, de los cuales veintisiete documentos completos fueron escogidos para la extracción de la información relevante y ajustada al objetivo planteado.

\section{Floraciones algales nocivas}

Las biotoxinas marinas son sustancias tóxicas acumuladas en los moluscos bivalvos por ingestión de plancton que contiene dichas toxinas. El aumento de la concentración de ciertos organismos componentes del plancton, bajo ciertas condiciones ambientales, produce un aumento explosivo de organismos fitoplanctónicos, que se conoce como florecimiento, floraciones algales, aguajes, o mareas rojas. La marea roja no es un peligro para la especie de moluscos bivalvos, pero sí lo es para las personas o consumidores potenciales. De los miles de tipos de mareas rojas, sólo un bajo porcentaje de ellas son dañinas para el ser humano $(10 \%$ aproximadamente del total) (11).

Las floraciones algales son del agua visibles a simple vista y debidas a proliferaciones de microorganismos planctónicos pigmentados (microalgas, ciliados, bacterias). En sentido amplio, el término «Floraciones Algales Nocivas» (o su acrónimo HAB, del inglés «Harmful Algal Blooms») ha sido acuñado por la COI (Comisión Oceanográfica Intergubernamental) de la UNESCO para designar las apariciones de un heterogéneo grupo de microorganismos que son percibidos como dañinos para el hombre por sus efectos adversos en la salud humana, en las explotaciones de acuicultura y turísticas de las zonas costeras, y en las poblaciones naturales de organismos marinos. Si bien el término se inspiró en las manchas de dinoflagelados planctónicos, hoy día se aplica a cualquier población microalgal, ya sea planctónica o bentónica, incluso aunque las concentraciones celulares no sean muy elevadas, siempre y cuando su aparición conlleve un efecto nocivo (12)

El fitoplancton marino está compuesto por una amplia variedad de especies de microalgas, entre las que se encuentran aquellas que forman florecimientos algales nocivos. Algunas son productoras de toxinas que provocan intoxicaciones en humanos, cuyos síntomas son principalmente neurológicos y gastrointestinales. De estos, los principales corresponden a la intoxicación paralizante, amnésica, diarreica, neurotóxica y ciguatera (13). 
De las 5000 especies de microalgas marinas descritas, aproximadamente 300 pueden producir floraciones algales y 75 han sido registradas como tóxicas, la mayoría de estas son dinoflagelados $(14,15)$.

\section{Organismo causante}

La intoxicación paralítica por el consumo de moluscos es un síndrome neurotóxico asociado al consumo de moluscos contaminados con toxinas paralíticas, que conforman un grupo de 21 tetrahidropurinas estrechamente relacionadas, con diferentes grados de toxicidad, siendo la más tóxica la saxitoxina, primera toxina caracterizada químicamente. Los dinoflagelados del género Alexandrium de zonas de clima tropical o templado son los principales responsables de la producción de estas toxinas (1).

Las especies del género Alexandrium (conocido como Gonyaulax o Protogonyaulax) identificadas como contaminantes de los mariscos son: Alexandrium tamarensis, A. minutum (syn. A. excavata), A. catenella, A. fraterculus, A. fundyense $y$ A. cohorticula. Otras especies de dinoflagelados, que también se identificaron como fuente de saxitoxina son Pyrodinium bahamense $y$ Gymnodinium catenatum. La toxicidad de los dinoflagelados varía según la mezcla de derivados de saxitoxina, cuya composición difiere según la especie que los produce y/o la región en que se presentan. Los moluscos asociados a casos de intoxicación paralitica son, principalmente, los moluscos bivalvos, es decir mejillones, almejas y en menor proporción ostras y vieiras (1).

\section{Composición química de la toxina}

Las toxinas paraliticas son un grupo de 21 tetrahidropurinas estrechamente relacionadas (16), (figura 1) cuya toxicidad dependerá del grupo químico que acompañe a los anillos hidropurínicos. La más tóxica es la saxitoxina. Las toxinas se pueden dividir en cuatro subgrupos:

i) carbamato (STX, neoSTX y las gonyautoxinas (GNTX1-4);

ii) N-sulfo-carbamoil (GNTX5-6, C1-4);

iii) decarbamoil (dc-) (dcSTX, dcneoSTX, dcGNTX1-4);

iv) compuestos de desoxidecarbamoil (do-) (doSTX, doneoSTX y doGNTX1).
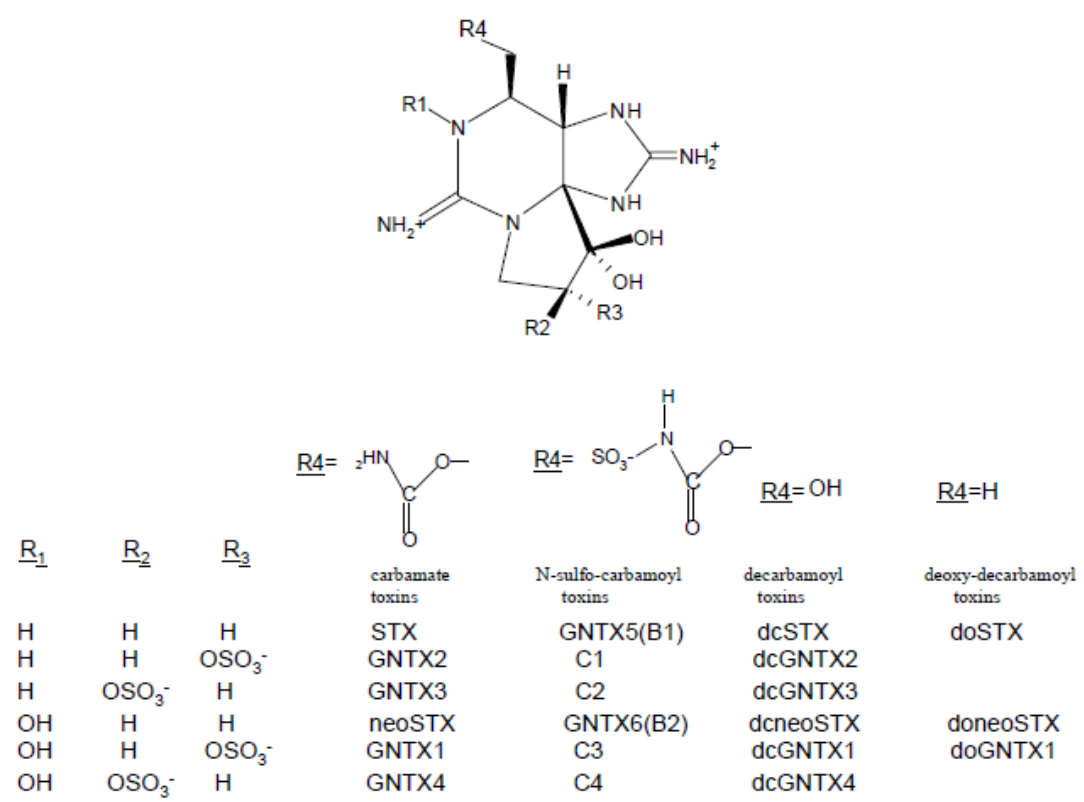

Figura 1. Estructura química de la STX y de otras toxinas paraliticas, (1). Fuente:estudio FAO: Alimentacion y Nutricion.2005-p:6 Se autoriza la reproducción y difusión del material contenido en este producto informativo para fines educativos $\mathrm{u}$ otros fines no comerciales sin previa autorización escrita de los titulares de los derechos de autor, siempre que se especifique claramente la fuente, copyright@fao.org 
El primer derivado cristalino de la saxitoxina se sintetizó en 1975. Esta se determinó mediante espectroscopia de resonancia magnética nuclear (RMN) y cristalografía de rayos X (1). La actividad tóxica reside en el grupo cetona hidratado o dihidroxi del anillo heterocíclico de cinco miembros, actividad que desaparece cuando estos grupos se transforman en monohidroxi por reducción catalítica con hidrógeno (1). La sustitución de la cadena secundaria con el grupo carbamoil en el anillo heterocíclico de seis miembros por un grupo hidroxilo resulta en una molécula con aproximadamente el 60 por ciento de la actividad tóxica original. Este grupo hidroxilo activo permite preparar distintos derivados de la saxitoxina. Las toxinas paraliticas son termoestables en $\mathrm{pH}$ ácido (salvo los componentes del N-sulfocarbamoil), pero inestables en condiciones alcalinas, oxidándose fácilmente (16).

\section{Epidemiología}

Los primeros casos de intoxicación paralitican por consumo de mariscos fueron reportados en el año 1793, por un marino llamado George Vancouver.en la actualidad en el período de 1973 a 1992, se han reportado 117 casos de intoxicación paralítica por moluscos, siendo la incidencia de esta intoxicación relativamente baja, puesto que desde 1940 se empezaron hacer controles de las aguas costeras donde se da el cultivo de los moluscos $(8,17)$

\section{Mecanismo de acción}

Las neurotoxinas tienen acción selectiva sobre el sistema nervioso periférico y sobre el músculo esquelético. Su mecanismo de acción es a través de la unión a la bomba de sodio voltaje-dependiente de las membranas celulares, a la cual bloquean, e interfieren en la transmisión y conductividad del impulso nervioso, produciendo así, una parálisis neuromuscular sin pérdida de conciencia. El periodo de incubación de los síntomas es corto: y varia de segundos a minutos (18).

Por otra parte, los músculo tienen una proteína llamada tropomiosina la cual puede causar alergia y dar como resultado un cuadro clínico de anafilaxia, que, si no responde al tratamiento, que asociado a la presencia de saxitoxina requiere atención médica y debe realizarse lo más rápido posible el apoyo cardiorespiratorio necesario (19).

\section{Manifestaciones clínicas}

Los síntomas pueden desarrollarse rápidamente, de 30 minutos a 2 horas después de la ingesta del molusco. La mayoría de los síntomas aparecen rápidamente, pueden durar varios días y son prácticamente invariables en todos los casos de intoxicación paralítica por consumo de moluscos (20). Comienzan con una sensación de hormigueo o quemazón de los labios, de la lengua, y de la cara con progresión gradual hacia el cuello, los brazos, la punta de los dedos de las manos, las piernas y los dedos de los pies. Luego ocurre un entumecimiento y los movimientos voluntarios resultan difíciles; puede aparecer afasia, sensación flotante/disociativa, salivación, cefalea, parestesias, sed, náuseas y vómitos. En el transcurso de dos a doce horas puede aparecer parálisis flácida e insuficiencia respiratoria y en ocasiones la muerte $(19,21,22)$.

Los síntomas van a depender de la concentración de la toxina y de la cantidad de moluscos contaminados ingeridos. Los niveles de toxina de 120 a $180 \mathrm{ug}$ pueden producir síntomas moderados, mientras que niveles entre 400 a 1060 ug pueden causar la muerte, que puede ocurrir 1 a 12 horas después de la ingesta (23). Se estima una mortalidad global del $10 \%$ y el pronóstico se relaciona con la rapidez en la instauración del mantenimiento ventilatorio adecuado (18). Si el paciente sobrevive 24 horas, el pronóstico es bueno, pero la debilidad muscular puede persistir por semanas (24).

\section{Diagnóstico}

El diagnóstico se basa en el antecedente de la reciente ingesta de comida marina, principalmente moluscos bivalvos (25). Se debe hacer diagnóstico diferencial con el botulismo, la ciguatera, y con intoxicaciones por insecticidas organofosforados y carbamatos (22).

\section{Tratamiento}


No hay un tratamiento ni antídoto específico, el tratamiento es sintomático y se basa en medidas de soporte hemodinámico y ventilatorio. Si el vómito no se ha presentado espontáneamente, hay que inducirlo o realizar un lavado gástrico para eliminar la fuente de toxina no absorbida. El carbón activado dede ser utilizado en la primera hora de la ingestión, no siendo de utilidad más allá de ese tiempo. Se debe hidratar al paciente, administrar diuréticos y bebidas alcalinas ricas en iones de sodio y potasio, ya que es la vía renal la principal vía de eliminación. En caso de insuficiencia respiratoria, se recurrirá a respiración mecánica. En casos severos se puede realizar hemoperfusión $(24,26)$.

\section{Medidas de control y prevención}

La principal medida preventiva es la inspección y monitoreo de las zonas de pesca y de los bancos de moluscos bivalvos para analizar la existencia de biotoxinas. Si se encuentran niveles altos de toxinas se interrumpe la captura comercial (27)

\section{DISCUSIÓN}

Aunque la intoxicación paralizante por el consumo de moluscos es un evento raro, la sintomatología es bastante alarmante y puede llevar a la muerte en pocas horas debido a la parálisis de los músculos respiratorios. La presencia de florecimientos algares nocivos, ha venido en aumento en los últimos años en Colombia, debido al aumento de los nutrientes y a la descarga de las aguas de lastre de los buques que entran a los principales puertos, que pueden traer consigo a los organismos productores. Esto conlleva a que se presenten cambios importantes en el ecosistema con la presencia de florecimientos de dinoflagelos tóxicos, poniendo en riesgo la salud de los habitantes y turistas de las zonas afectadas.

En las costas colombianas hasta ahora no se han registrado casos de intoxicaciones y muertes en humanos; sin embargo, cabe la posibilidad de que puedan presentarse, ya que han sido registrados eventos tóxicos en las costas de algunos países de Centroamérica y Sudamérica como Ecuador, Perú y Chile. En estudios realizados en muestras de agua durante las pocas mareas rojas presentadas en el país, se ha detectado la presencia de la especie
Alexandrium causante de la intoxicación paralítica por moluscos $(9,10)$.

A pesar de que en Europa, América del norte, algunos países de Sur y Centroamérica, África y Oceanía tienen reglamentación en cuanto a programas de control para verificar la presencia de organismos productores de toxinas, en Colombia aún no existe regulación ni programas de monitoreo y las pocas investigaciones realizadas corresponden a iniciativas aisladas de grupos de investigadores de universidades y centros de investigaciones marinas (10).

Se hace necesario que se definan políticas claras en cuanto a la regulación de la presencia de dinoflagelados en aguas costeras, así como la capacitación al personal de salud para hacer el diagnóstico adecuado y a tiempo previniendo las complicaciones fatales por la intoxicación asociada a la saxitoxina.

\section{CONCLUSIÓN}

Por ser las microalgas el primer nivel trófico alimenticio acuático, es necesario implementar estrategias de manejo costero integrado que ayuden a estar vigilantes a los eventos de mareas rojas e identificar los problemas de valoración ambiental.

CONFLICTO DE INTERÉS: ninguno que declarar.

\section{REFERENCIAS}

1. Fao. Biotoxinas marinas. Food Sci Technol Int. 2005;2(1):64.

2. Costa PR, Botelho MJ, Rodrigues SM. Accumulation of paralytic shellfish toxins in digestive gland of Octopus vulgaris during bloom events including the dinoflagellate Gymnodinium catenatum. Vol. 58, Marine Pollution Bulletin. 2009.

3. Finnis S, Krstic N, McIntyre L, Nelson TA, Henderson SB. Spatiotemporal patterns of paralytic shellfish toxins and their relationships with environmental variables in British Columbia, Canada from 2002 to 
2012. Environ Res. 2017;156:190-200.

4. Handy SM, Yakes BJ, DeGrasse JA, Campbell K, Elliott CT, Kanyuck KM, et al. First report of the use of a saxitoxin-protein conjugate to develop a DNA aptamer to a small molecule toxin. Toxicon. 2013;61:30-7.

5. Liu Y, Yu R-C, Kong F-Z, Chen Z-F, Dai L, Gao Y, et al. Paralytic shellfish toxins in phytoplankton and shellfish samples collected from the Bohai Sea, China. Mar Pollut Bull. 2017;115(1):324-31.

6. Vale P. Fate of benzoate paralytic shellfish poisoning toxins from Gymnodinium catenatum in shellfish and fish detected by pre-column oxidation and liquid chromatography with fluorescence detection. J Chromatogr A. 2008 May;1190(1-2):191-7.

7. Fleming LE, Broad K, Clement A, Dewailly E, Elmir S, Knap A, et al. Oceans and human health: Emerging public health risks in the marine environment. Mar Pollut Bull. 2006 Jan;53(10-12):545-60.

8. Gessner BD, Middaugh JP. Paralytic Shellfish Poisoning in Alaska: A 20-Year Retrospective Analysis. Am J Epidemiol. 1995 Apr 15;141(8):76670.

9. Del FYA, Arteaga E, Victoria L, Trujillo P. Distribución, frecuencia y abundancia del fitoplacton potencialmente toxígeno en la cuenca pacífica colombiana. Boletín Investig Mar y Costeras. 2016;45(1):135-48.

10. Mancera Pineda JE, Gavito B, Arencibia Caraballo G. Floraciones Algales Nocivas, Intoxicación por Microalgas e Impactos en el Desarrollo Regional: el Caso de San Andrés Isla, Caribe Colombiano. Cuad del Caribe. 2010;13:46-62.

11. Del T, Salazar CM. Las biotoxinas marinas en los alimentos Biotoxines marines in food. Rev.Per Obst Enf.2006; 2(2):1-11

12. Sar E, Ferrario M, Reguera B. Floraciones Algales Nocivas en el Cono Sur Americano. Madrid: Instituto Español de Oceanografia; 2002. 311 p.

13. Alonso-Rodríguez $\mathrm{R}$, Mendoza-Amézquita $\mathrm{E}$, Velásquez-López SA, Seim JA, Martínez-Rodríguez
VM. Florecimientos algales nocivos producidos por Pyrodinium bahamense en Oaxaca, Mexico (20092010). Salud Publica Mex. 2015;57(4):343-51.

14. Baylón M, Sánchez S, Bárcena V, López J, Mamani E, Maribel Baylón E, et al. First record of potentially toxic dinoflagellate, Alexandrium minutum Halim 1960, from Peruvian coastal. Rev Peru Biol. 2015;22(221):113-8.

15. Torres G. Eventos de mareas rojas: estrategias de manejo preventivas en Ecuador. Universidad de Guayaquil; 2011.Rev Acta Oceanografica del Pacifico.2015;20(1)89-99.

16. Chegini S, Austin SJ, Metcalfe DD. Seafood Toxins. Foodborne Diseases. 214AD. 518-534 p.

17. Finnis S, Krstic N, McIntyre L, Nelson TA, Henderson SB. Spatiotemporal patterns of paralytic shellfish toxins and their relationships with environmental variables in British Columbia, Canada from 2002 to 2012. Environ Res. 2017; 156(January):190-200.

18. Franquelo Cabrera F, Domínguez Pión F, Cruz-conde De Boom R, Tellez Andrade A, Quesada García G. Intoxicación paralítica por consumo de marisco. Emergencias. 2009;21:306-8.

19. Tania Guerrero Marin, A. C. Ron Aguirre, E. Saleme Cruz, H. Montiel Falcon L Dominguez Gordillo. Intoxicación paralítica por ingesta de moluscos. Reporte de caso. An Médicos. 2013;58(3):5-8.

20. Rodriguez A. Evaluación de los resultados del laboratorio de toxinas marinas durante el periodo 2008-2012, en el marco del programa de vigilancia y control de marea roja en la provincia de río negro.Repositorio Univ Nac La Pampa. 2014; p 1-54

21. De CO de la RN, (RENAPRA) P de A. Enfermedades transmitidas por alimentos. Red Nacional de Proteccion de Alimentos. Ficha Técnica N5. 2009; p2-5

22. Alvarado Noriega JR. Neurotoxicidad por intoxicación alimentaria: toxinoinfección por consumo de mariscos. Boletin Médico de Postgrado. Vol. 26 Núm.1. Enero 2010 p. 26-35.

23. Ching PK, Ventura RJ, de los Reyes VC, Sucaldito 
MN, Tayag E. Lethal paralytic shellfish poisoning from consumption of green mussel broth, Western Samar, Philippines, August 2013. West Pacific Surveill Response J. 2015;6(2):22-6.

24. Sánchez Flores HE. Envenenamiento paralizante severo por consumo de moluscos. Reporte de un caso. Arch Med Urgenc México. 2011;3:30-3.

25. Isbister GK, Kiernan MC. Neurotoxic marine poisoning. Lancet Neurol. 2005 Apr;4(4):219-28.

26. Leiva Hidalgo J, Soto Flores S. Las mareas rojas en Costa Rica. Rev Med Costa Rica. 2010;77(595):4336.

27. Poot Delgado CA. Normativa aplicada al manejo de moluscos bivalvos expuestos a mareas rojas. In: XXI Congreso Ciencia y Tecnologia del Mar. Cozumel Quintana Roo; 2014. 